\title{
Research Paper: Prevalence of Comorbidity Behavioral Disorders in Children With Attention Deficit Hyperactivity
}

\author{
Mahsa Sattari ${ }^{1},{ }^{*}$ Seyed Ali Hosseini ${ }^{2}$, Mehdi Rassafiani ${ }^{2}$, Mohammad Javad Mahmoudi Gharaei ${ }^{3}$, Akbar Biglarian ${ }^{4}$, Najmeh \\ Tarkesh Esfahani ${ }^{4}$
}

1. Department of Occupational Therapy, School of Paramedical \& Health, Zanjan University of Medical Sciences, Zanjan, Iran

2. Department of Occupational Therapy, University of Social Welfare and Rehabilitation Sciences, Tehran, Iran

3. Department of Psychology, School of Medicine, Tehran University of Medical Sciences, Tehran, Iran.

4. Department of Biostatistics, University of Social Welfare and Rehabilitation Sciences, Tehran, Iran.

Crtation: Sattari M, Hosseini SA, Rassafiani M, Mahmoudi Gharaei MJ, Biglarian A, Tarkesh Esfahani N. [Prevalence of Comorbidity Behavioral Disorders in Children With Attention Deficit Hyperactivity (Persian)]. Archives of Rehabilitation. 2017; 18(1):25-32. https://doi.org/10.21859/jrehab-180125

: https://doi.org/10.21859/jrehab-180125

Received: 19 Oct. 2016 Accepted: 29 Jan. 2017

Keywords: Attention deficit hyperactivity disorder, Comorbidity, Behaviora problems

\section{ABSTRACT}

Objective Attention Deficit Hyperactivity Disorder (ADHD) is the most common psychiatric disorders among children referred to treatment centers that caused problems in their personal and social lives. Thus, this study aimed to investigate the prevalence of behavioral problems in children with ADHD. Methods \& Materials This descriptive-analytical study included 278 children (aged 7-11 years) with ADHD who were referred to the Children's Medical Center and Clinic of Psychiatry, Atiyeh during 30-03-2012 to 20-04-2012. The behavioral problems of children who were diagnosed with ADHD by psychiatrists were assessed using Child Symptom Inventory (CSI-4). SPSS19 was used to describe and analyze data.

Results Based on the results, most visitors were boys, and AD in the girls and combined ADHD in the boys had the highest frequency. No significant relationship was found between gender and type of disorder. Most of the children reported only one behavioral disorder such as confrontational behavior, specific phobia, generalized anxiety disorder, separation anxiety and dysthymia.

Conclusion The prevalence of inventory disorders in the three types of ADHD varied according to age and sex. Statistically significant differences were observed between the three types of ADHD, confrontational disorder (4000.0), and anxiety (0.02).

\section{* Corresponding Author:}

Seyed Ali Hosseini, PhD

Address: Department of Occupational Therapy, University of Social Welfare and Rehabilitation Sciences, Evin, Daneshjou Blvd., Koudakyar St., Tehran, Iran. Tel: +98 (21) 22180036

E-Mail: alihosse@gmail.com 


\title{
بررسى ميزان همبودى اختلالات رفتارى در كودكان مبتلا به نقص توجه و بيشفعالى
}

مهسا ستارى"، "سيد على حسينى"، مهدى رصافيانى"، محمدجواد محمودى قرايى"، اكبر بيكلريان"، نجمه تركش اصفهانى"

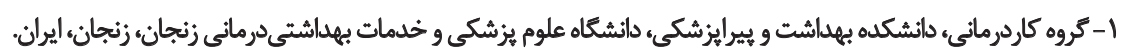

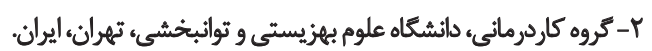

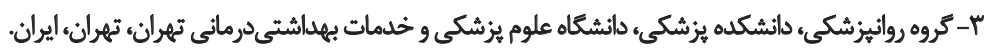

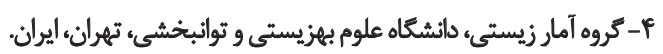

\begin{abstract}
(S)

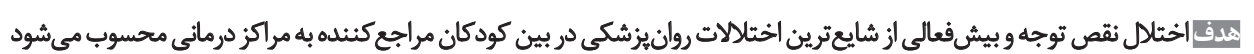

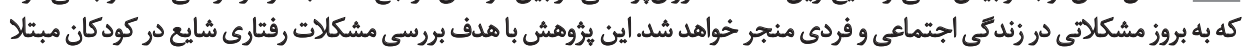

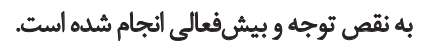

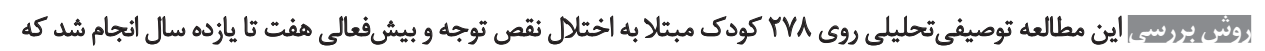

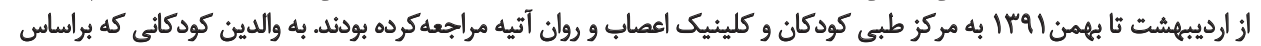

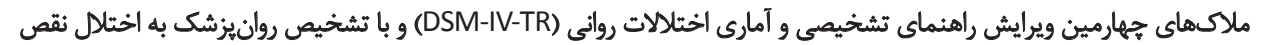

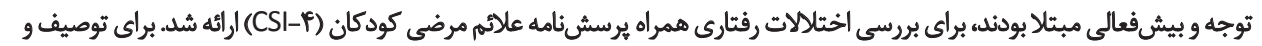

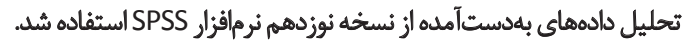

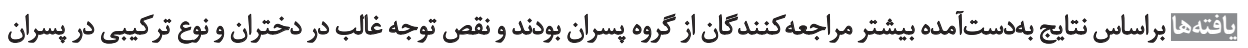

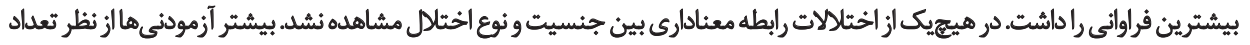

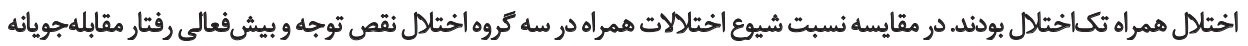

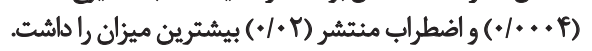

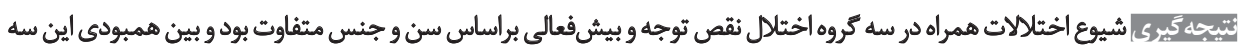

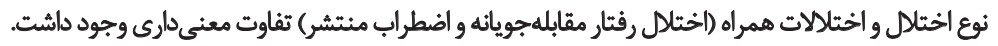

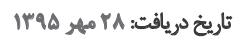

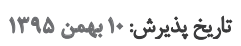

\author{
كليدوارثها: \\ نقص توجها ور

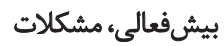 \\ رفتارى، همبودى
}

r. عمدتاً بيش فعال و تكانشكَر. ه نسبت شيوع اين اختلال در بسران

مقلمه

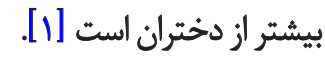

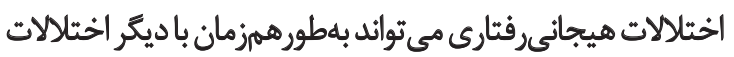

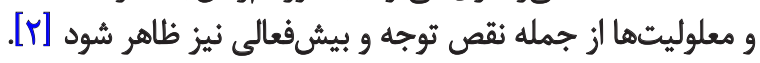

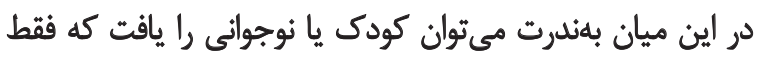

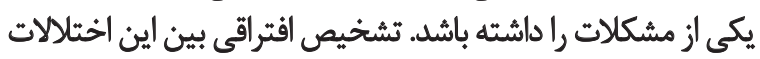

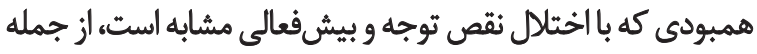

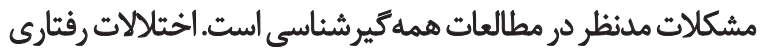

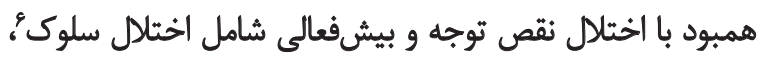

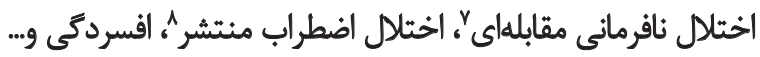

اختلال نقص توجه و بيشفعالى (ADHD) ' يكى از تستردهترين

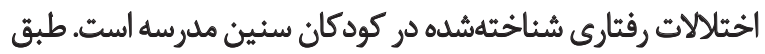

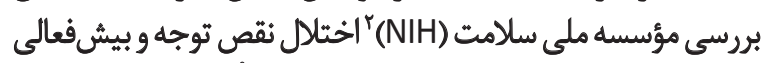

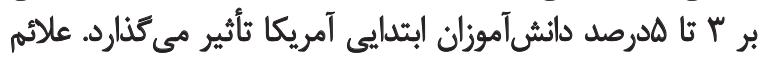

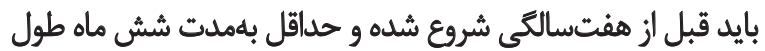

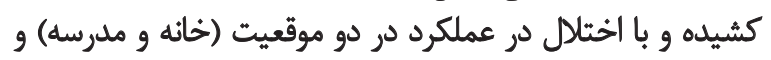

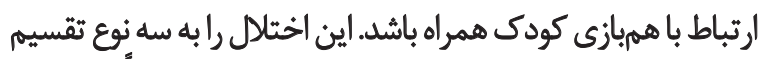

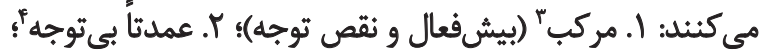

5. ADHD-PH/I

6. Conductive Disorder (CD)

1. Attention Deficit Hyperactivity Disorder (ADHD)

2. National Institutes of Health

3. ADHD-C

4. ADHD-PI

8. Generalized Anxiety Disorder (GAD)

: نويسنده مسئول:

دكتر سيد علي حسيني

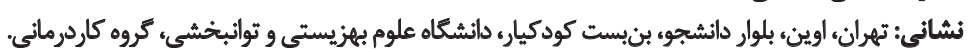

+ تلفن:

رايانامه: 


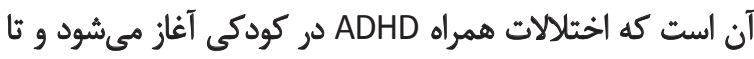

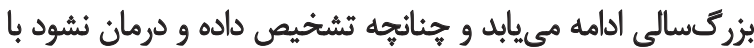

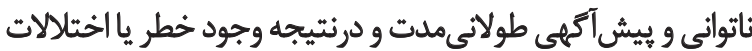

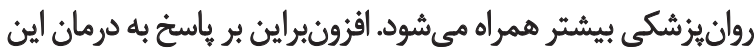

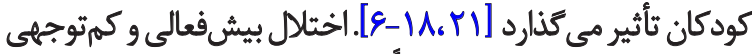

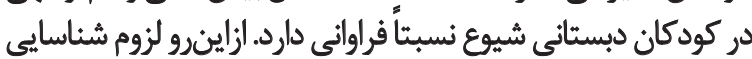

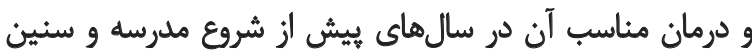

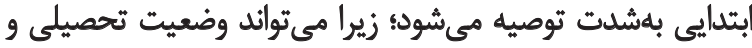

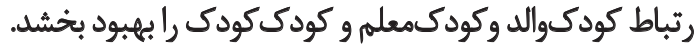
بهدليل تنوع مشكلات مربوط به ADHD مسلماً امكان آن وجود

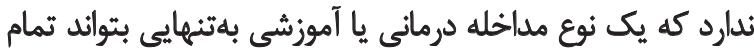

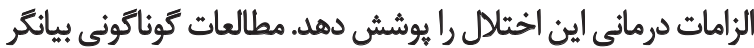

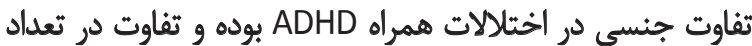

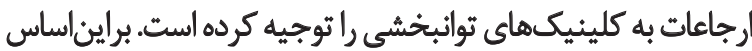

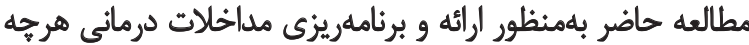

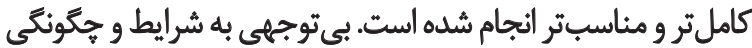

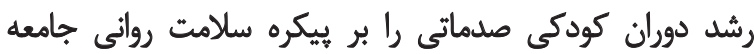

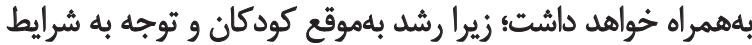

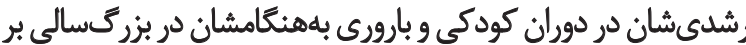

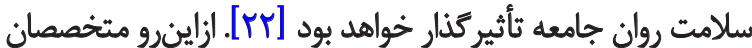

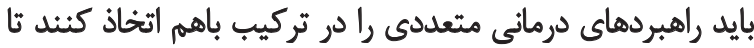

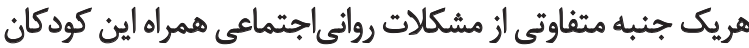

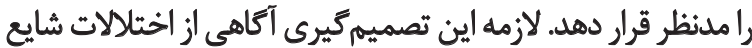

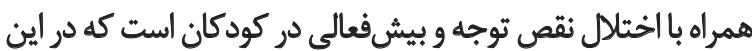
يروهش به آن يرداخته شده است.

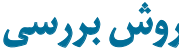

جامعه و نُمونه و ووش نمونه كيّي

در اين مطالعه توصيفى تحليلى جامعه آمارى تحقيق سيصد نفر از

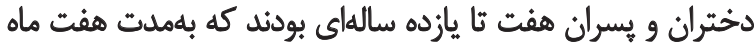

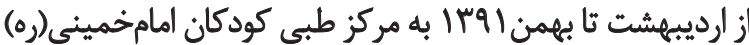

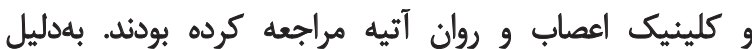

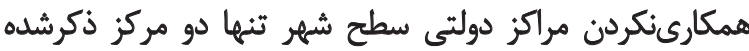

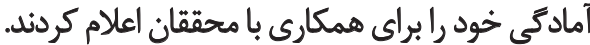

حجم نمونه براساس جدول كرجسى موركان، بهصورتى تعيين

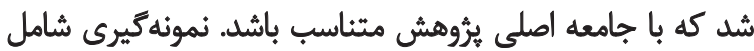

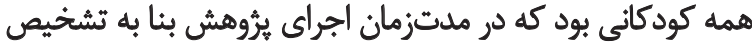

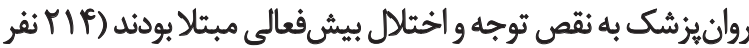

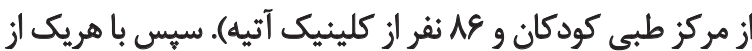

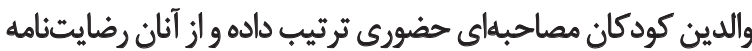

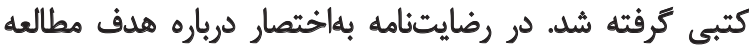
توضيح داده و بر محرمانهماندن ثأكيد شده بودي
است. در بسيارى از موارد اين اختلالات در كودك تا سن مدرسه

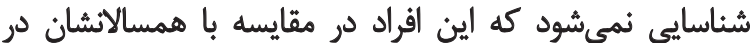

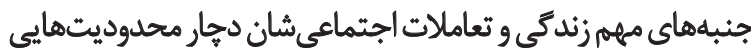

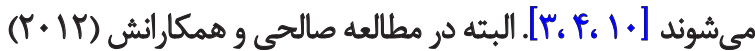
تفاوت معنادارى بين همبودي ADHD و اختيلالات همراه مانئد

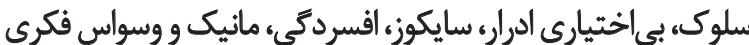

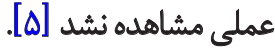

اختلالات رفتارىهيجانى شرايطى است كه در آن باسخهاي

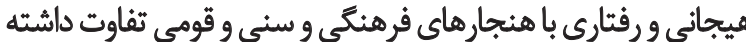

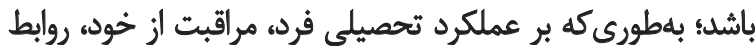

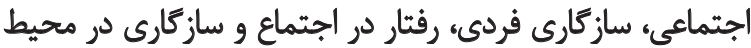

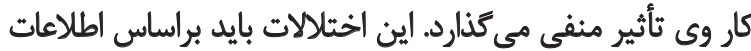

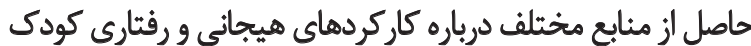
شناسايى شود و حداقل در دو موقعيت متفاوت (مدرسه و وخانيانه ) بروز

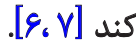

تاكنون طبقهبندىهاى مختلفى از اختلالات رفتارى ارائه شده

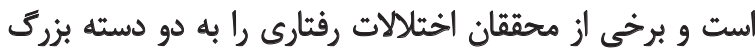

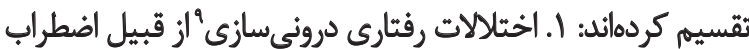

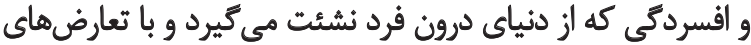

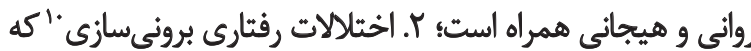

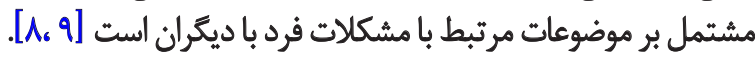

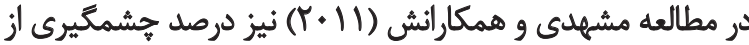

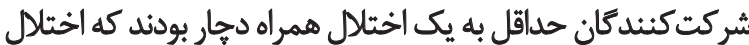

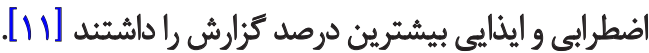
همجُنين اثرهاى منفى اختلالات رفتارى بر عملكرد

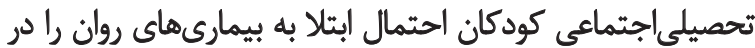

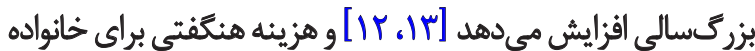

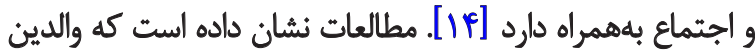
اين كودكان بهدليل نشناختن دقيق اختالل و نداشتن آكاهي لازئ

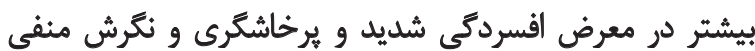

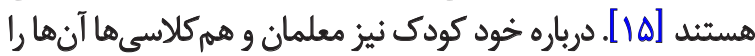

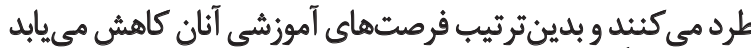

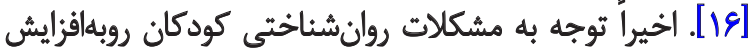

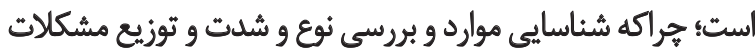

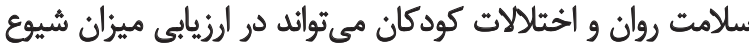

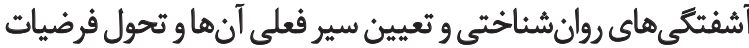
سببشناسى آنها استفاده شود [1/11].

درباره همراهى ADHD با اختلالات روانيزشكى تحقيقات

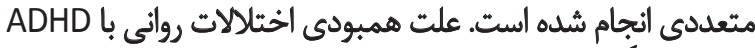
هنوز كاملاً شناخته نشده است؛ علام اما اطلاعات دردسترس كوياى روانى 


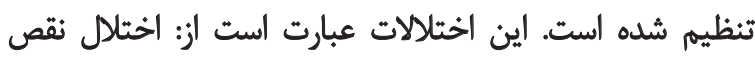

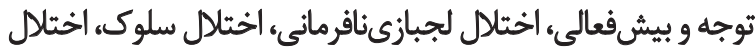

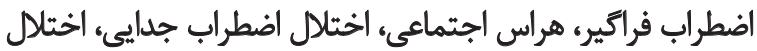
وسواس فكرىعملى، هراس خاص، اختلال افسردئى اساسى، اختي، اختلال

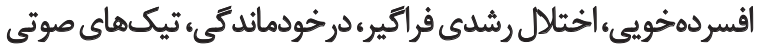
و حركتى، اختلال استرس يس از سانحه و اختلالات دفع. ملاك انتخاب طبقات تشخيصى مطرحشده در يرسش نام كامه علائم

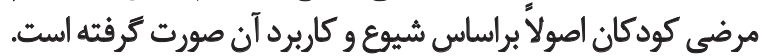

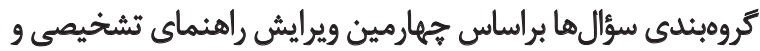

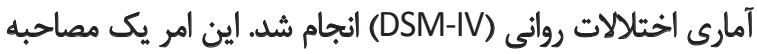

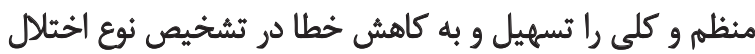

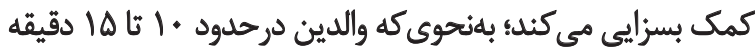

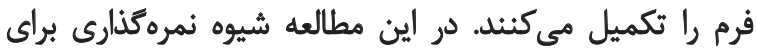

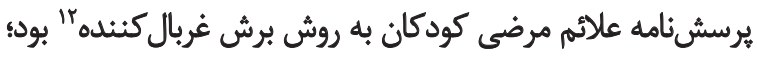

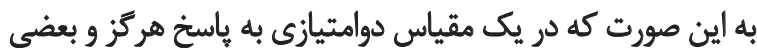

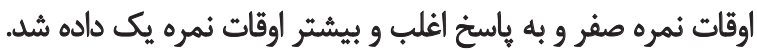

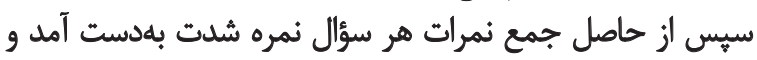

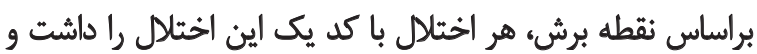

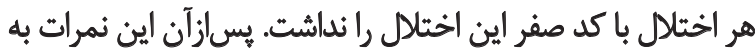

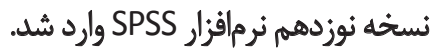

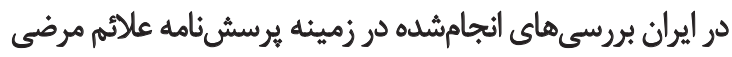

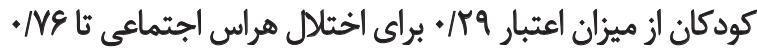

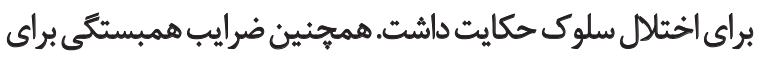

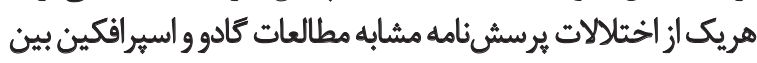

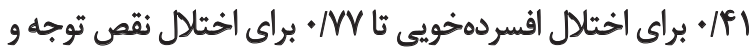

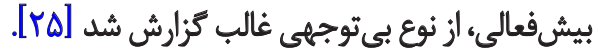

12. The screening cut of score method
بهوسيله يرسشئامه اطلاعات لازم براي انجام برؤوهش مانند سن

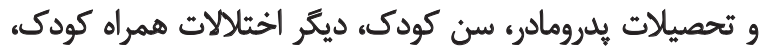

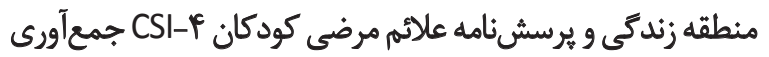

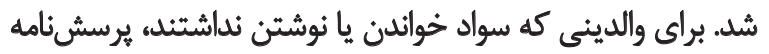

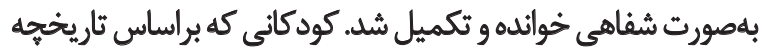

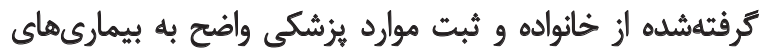

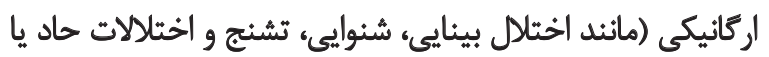

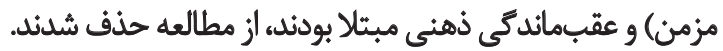
با آزمون ريون سياهوسفيد متغيرهاى زمينهاى از قبيل ونيل وضعيت

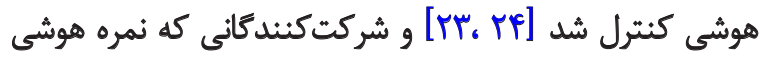

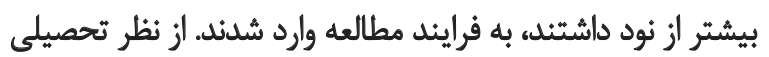

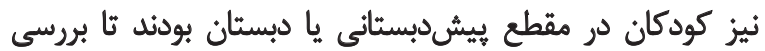

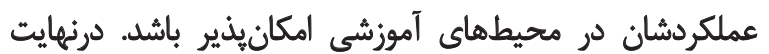

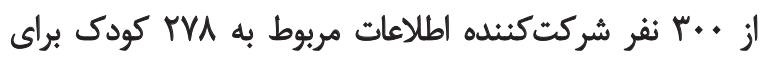

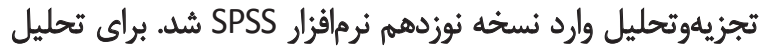

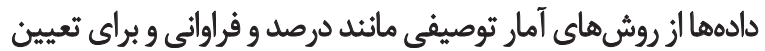

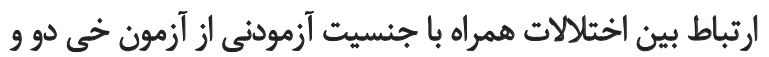

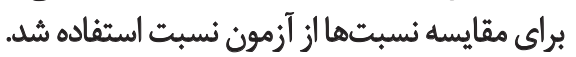

\section{"يرسش نامه علاثم مرضى كودكان FSI-F" (نسخه والد)} اين برسشنامه مقياس درجهبندى رفتار محسوب مى رئود كه

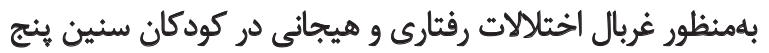

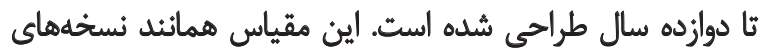

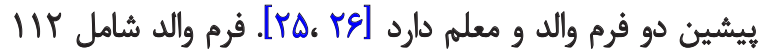

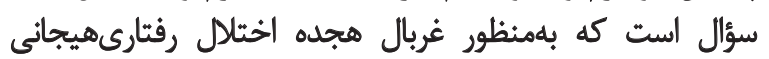

11. Children Symptom Inventory-4 (CSI-4)

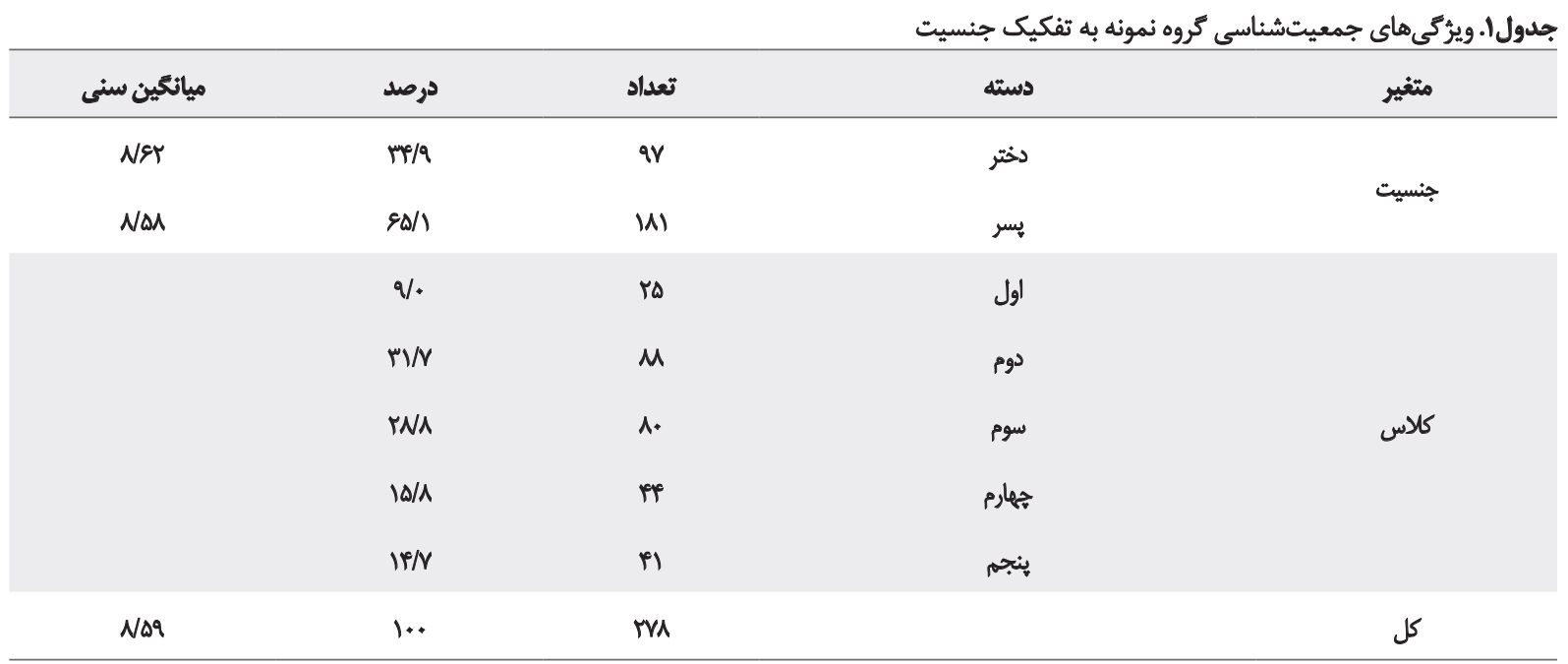


نظر تعداد اختلالات همراه مشاهده نشد (ه •.1P). در جدول شماره باين موضوع بررسى شده است كه آيانسبتهاى

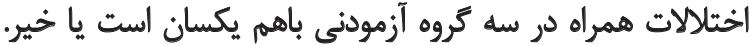

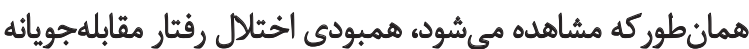

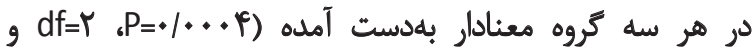

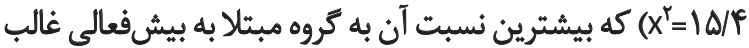

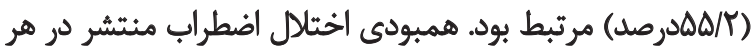

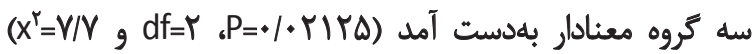

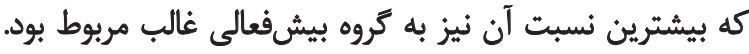

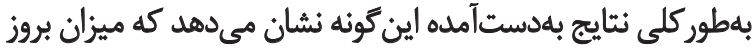

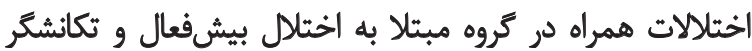
(ADHD-PH/l)

ث

با توجه به يافتهاى بهدستآمده از يُروهش حاضر بلنظر معرسد

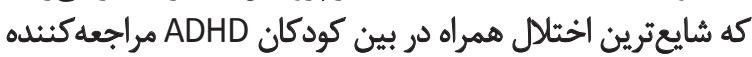

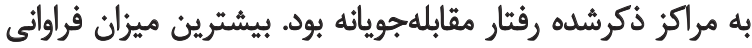

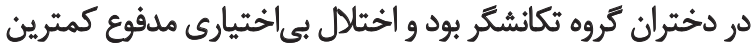

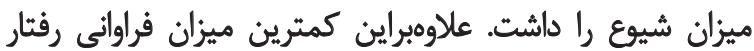

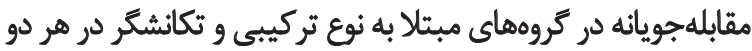

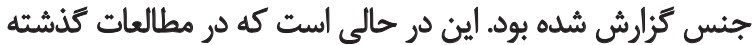

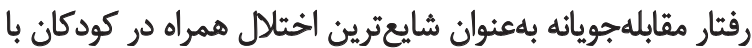
ADHD-C

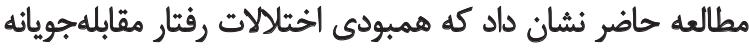

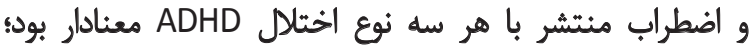
درصورتى كه در ديكر اختلالات همراه رابطه معنادارى مشاهده نشد. نتايج بهدست آمده از مطالعه حاضر با هطالعه صالحي و همكارانش

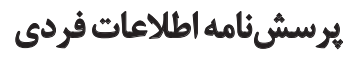

يثروهشكَران اين برسشنامه را بهمنظور بروسى شرايط كودى از

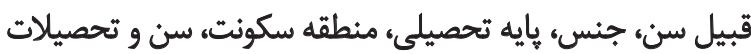
والدين و درآمدشان طراحى كرده است تصديل

ياقتهنها

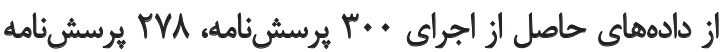

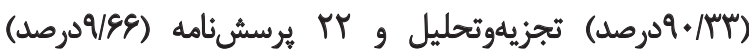

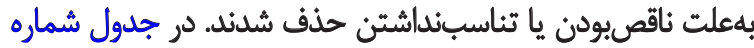

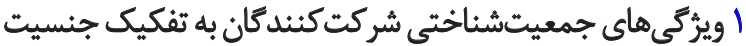

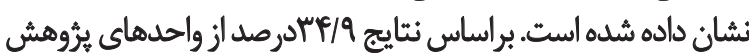

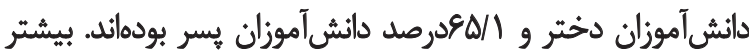

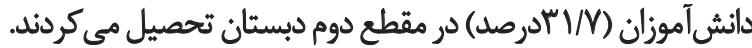

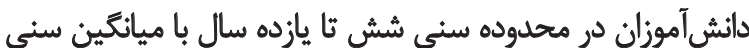

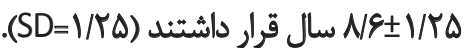

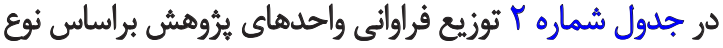

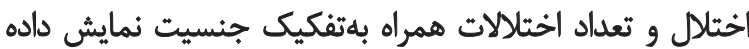

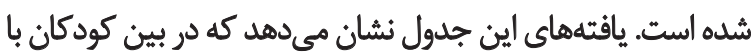

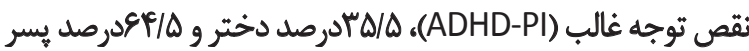

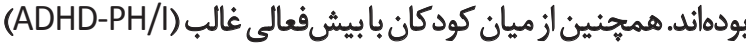

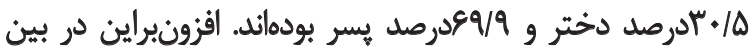

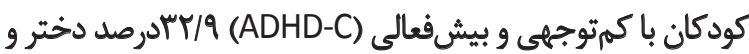

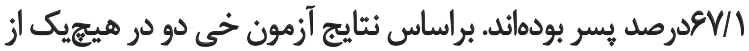

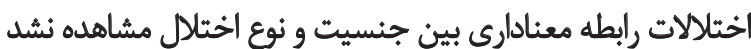

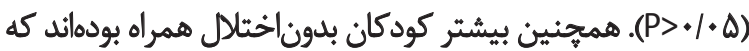

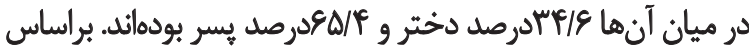

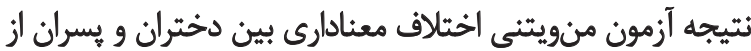

جدول ז. توزيع فراوائى واحدهاي يرؤهش براساس نوع اختلال و تعداد اختلالات همراه بهتفكيك جنسيت

\begin{tabular}{|c|c|c|c|c|c|c|}
\hline مقدار احتمال & ملاكى آزمون & تعداد (درصد) & تعداد (درصد) & تعداد (درصد) & اختثلال همراه & \\
\hline.$/ \Delta \Delta A$ &.$/ m T^{*}$ & $\operatorname{mer}(1 . .1)$. & $1 \Delta E(g / \Delta)$ & $N \in(r \Delta / \Delta)$ & نقصص توجه غالب & \\
\hline .1119 & r/eme & $\mid+1(1.010)$ & $Q(E q / 9)$ & $\operatorname{Rr}(r \cdot / \Delta)$ & ييش فُعالى غالب & نوع اختلال \\
\hline $.1+\Delta V$ & r/grts & $\operatorname{reg}(1.01)$. & $19 \Delta(9 \vee /)$ & 1) $(r T / Q)$ & كمتوجهى و بيش فعالى & \\
\hline \multirow{7}{*}{ سוז/. } & \multirow{7}{*}{. 1 ATES"* } & $A 1(1 . .1 \%)$ & $\Delta \Psi(F \Delta / \tau)$ & TA(MT/8) & بلدوناختلالل & \multirow{7}{*}{ تعداد الختالالات همراه } \\
\hline & & $\operatorname{\Delta r}(1 . .1 \cdot)$ & $r \cdot(M=/ 1)$ & $I f(r \Delta / q)$ & يك امختهالال & \\
\hline & & $r g(1.0 \%)$ & $r Q(q q / F)$ & $11(r+/ 8)$ & دو اختّلال & \\
\hline & & $m(1.0 \%)$ & $18(F+/ 1)$ & $19(\Delta \Delta / 9)$ & سه اختلال & \\
\hline & & $\mid V(1 . .1 \%)$ & $1 r(v-18)$ & $\Delta(r Q P r)$ & جهار إختلال & \\
\hline & & $19(1+.1 \%)$ & IO(YNQ) & $F(r) / M)$ & ينج اختلال & \\
\hline & & $M(1+0 / \%)$ & $r(\Delta \& / A)$ & $19(Y r / T)$ & شش اختلال و بيشتر & \\
\hline
\end{tabular}


جدول r. مقايسه نسبتهاى بروز اختلالات همراه در سه تروه آزمودنى

\begin{tabular}{|c|c|c|c|c|c|}
\hline مقدار احتمال & ملاك آزمون & بيشداد (دالى غالب) & تعداد (درصى غالب) & تعداد (دربب & اختلال همراه \\
\hline$+10++$ & $10 /=0$ & $n+(\Delta \Delta / T)$ & 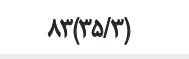 & $9 \cdot(T V / 8)$ & رفثار مقابلهجويانه \\
\hline . MIEF & $p / r q$ & $19(1 \% / 1)$ & $19(N \cdot)$. & $r \cdot\left(N r_{0}\right)$ & اختلال سلوى \\
\hline \%rITS* & $V / N$. & $\Delta A(P T / T)$ & $R \cdot(r q M)$ & $V \Delta(r / M)$ & اضطر|ب متثتر \\
\hline $.10 .+1$ & 1/ז1 & rA(YS/) & $p q(T \cdot / A)$ & $\Delta T(Y Y / M)$ & هراس خاص \\
\hline +/PN1Q & $1 / F$ & $\mid M(1+/ 4)$ & $r r(\cdot q / T)$ & $r q(1 . / 1)$ & وسواس فكرى \\
\hline -NETI &.$/ \Delta T^{\circ}$ & $I Y(I T / 8)$ & $r(1 \cdot \pi)$ & $r e(1 \cdot 14)$ & وسواس عملى \\
\hline . rqur & $r / F)$ & $M(I V /)$ & TA(IV/9) & $r q(1 T / 1)$ & هراس هيس الز سانحه \\
\hline+18799 & $\cdot 1 N$ & $r M(Y \circ / A)$ & $F \cdot(I V / *)$ & $F \triangle(I N A)$ & تيك حركتى \\
\hline.$/ 8 \cdot \Delta V$ & $1 /$. & $I V(I Y / P)$ & $M(Q / M)$ & $r e(1 . / 1)$ & تيك صوتى \\
\hline.$/ 018 A$ & $1 / M$ & $V(\Delta / T)$ & $V(Y / 9)$ & $A(M / Y)$ & اختالال خلقى \\
\hline . Rreq. & $r / Y \wedge$ & $\operatorname{Pr}(\Psi \mathbb{M} / T)$ & $\Delta E(T / / A)$ & $\Delta q(Y Y / g)$ & افسردخويى \\
\hline 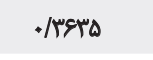 & $r / \cdot r$ & $r r(1 / / 4)$ & $r Y(1 Y / P)$ & rq(IT/I) & الوتيسم \\
\hline -195A9 & .1 .8 & $r \otimes(I N F)$ & $P r(I V / A)$ & $P r(I V / \theta)$ & جمعيت هراسى \\
\hline . IOVIY & $1 / 11$ & $M(M+/ 9)$ & $P q(T \cdot / A)$ & PAY./.) & اضطراب جدايى \\
\hline$+/ 9 T \Delta V$ & .110 & $1 F(1 . / 4)$ & $r(q / V)$ & $\pi(q / Y)$ & شبادرارى \\
\hline .MMTI & . Iar & $g(\varphi / 4)$ & $(r / \mu) \mu$ & $V(T / 9)$ & بىاختيارى هدفوع \\
\hline
\end{tabular}

كروه مبئلابه اختلال بيش فيشال وتكائشكر (ADHD-PH/I) در مقايسه

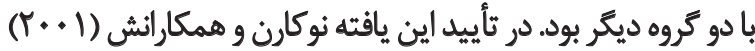

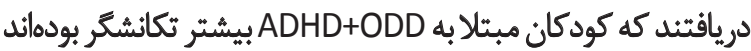

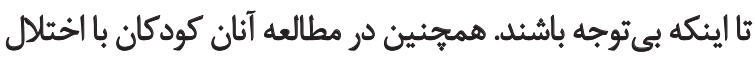

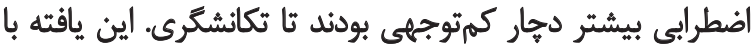
ياقتههاي حاصل از مطالعه حاضر همخوان نبود [IV، YV]

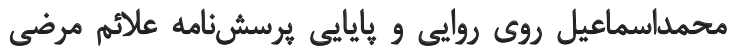

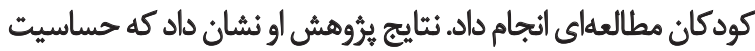

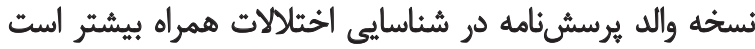

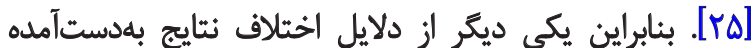

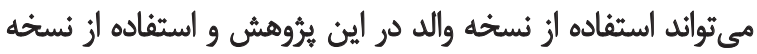

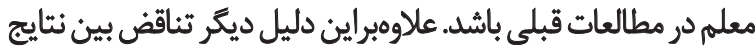

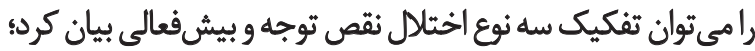

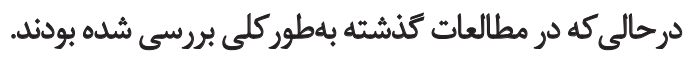

\section{نتيجنَّيرى}

يافتههاى يروهش نشان داد اختالل ADHD در بسيارى از موارد با

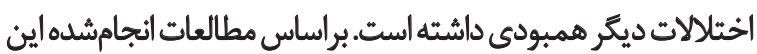
امر مىتواند بيه مقاومت بيشتر به روند درمانى و مواجهه بـ با مشكلات

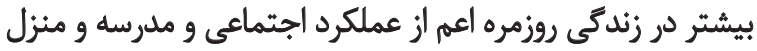
منجر شود. ازاينرو توصيه مي شود اختيلالات همر اه دقيقتر شئاسايى

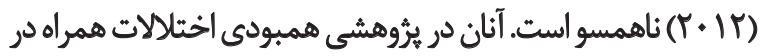

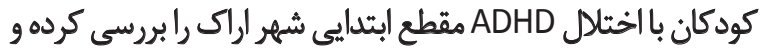

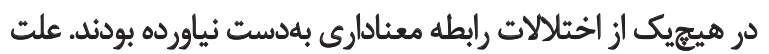

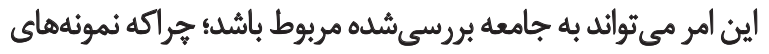

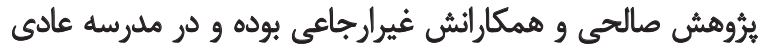

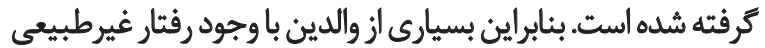

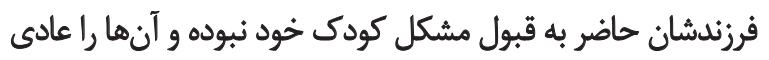

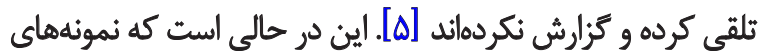

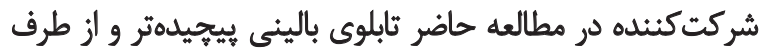

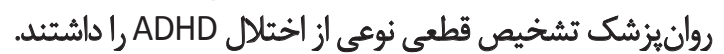

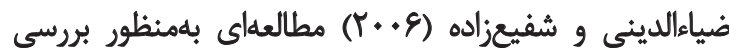

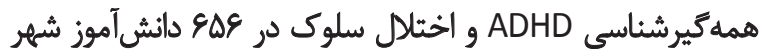

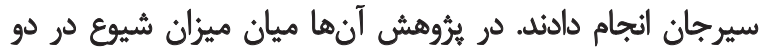

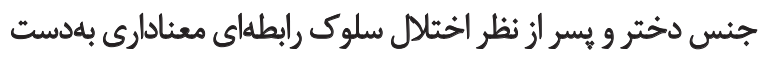

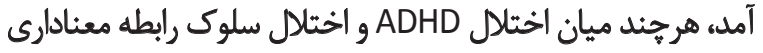

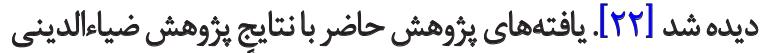

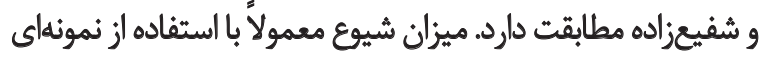

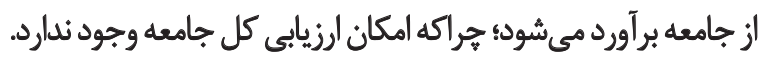

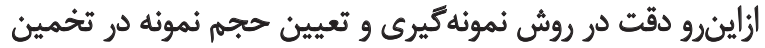

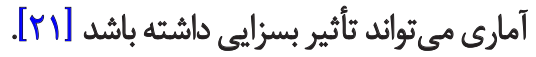
يافته ديگر يثوهش حاضر فراوانى ميزان نافرمانى مقابلهجويائه در 


\section{References}

[1] Sadock BJ, Sadock VA. Kaplan and Sadock's pocket handbook of clinical psychiatry. Philadelphia: Lippincott Williams \& Wilkins; 2010

[2] Tankersley M, Landrum TJ. Comorbidity of emotional and behavioral disorders. In: Lioyd JW, Kameenui EJ, Chard D, editors. Issues in Educating Students With Disabilities. Mahwah: Erlbaum; 1997.

[3] Walker HM, Forness SR, Kauffman JM, Epstein MH, Gresham FM, Nelson CM, et al. Macro-social validation: Referencing outcomes in behavioral disorders to societal issues and problems. Behavioral Disorders. 1998; 24(1):7-18.

[4] Yoosefi F. [Standardization of Rotter's scale to verify the behavioral and emotional problems in girls and boys of Shiraz (Persian)]. Journal of Social Sciences \& Humanities of Shiraz University. 1998; (26):171-94.

[5] Salehi B, Ebrahimi S, Moradi S. [Comparing psychiatric comorbidity in female and male primary school children with attention deficit hyperactivity disorder, Arak, Iran (Persian)]. Journal of Hormozgan University of Medical Sciences. 2012; 16(1):49-59.

[6] Ghobari Bonab B, Parand A, Hossein Khanzade Firoozjah AA, Movalleli G, Nemati S. [Evaluation of the onset behavior problems among primary school students in Tehran (Persian)]. Journal of Exceptional Children. 2009; 9(3):223-38.

[7] Al-Yagon M. Comorbid LD and ADHD in childhood: soci oemotional and behavioural adjustment and parents' positive and negative affect. European Journal of Special Needs Education. 2009; 24(4):371-91. doi: 10.1080/08856250903223054

[8] Achenbach TM, Howell CT, Quay HC, Conners CK, Bates JE. National survey of problems and competencies among four-to sixteen-year-olds: Parents' reports for normative and clinical samples. Monographs of the Society for Research in Child Development. 1991; 56(3):1-130. doi: 10.2307/1166156

[9] Gadow KD, DeVincent CJ, Schneider J. Comparative study of children with ADHD only, autism spectrum disorder+ ADHD, and chronic multiple tic disorder+ ADHD. Journal of Attention Disorders. 2009; 12(5):474-85. doi: 10.1177/1087054708320404

[10] Molagholamreza Tabasi F, Aliabadi F, Alizade Zarei M, Qorban M, Rostami R. Survey of behavioral problems and sensory processing in children with Attention Deficit/Hyperactivity Disorder (ADHD). Iranian Rehabilitation Journal. 2016; 61-70.

[11] Mashhadi A, Soltanifar A, Moharreri F, Noferasati F. [Prevalence of comorbid psychiatric disorders and its effect on the response to methylphenidate in children with ADHD (Persian)]. Pajoohande. 2011; 16(4):162-8.

[12] Shafaat A, Tirgari-Seraj A, Daneshpoor S M M, Hajian M, Khademloo M. [Prevalence of Attention Deficit Hyper Activity Disorder in high-school students of Sari, Iran. (Persian)]. Journal of Mazandaran University of Medical Sciences 2013; 23(104):12 18.

[13] Pandina G, Bilder R, Harvey P, Keefe R, Aman M, Gharabawi G. Risperidone and cognitive function in children with disruptive behavior disorders. Biological Psychiatry. 2007; 62(3):226-34. doi: 10.1016/j.biopsych.2006.09.036
و براي درمان مناسب آن در روند درماني مراكز و مدارس اقدام شود.

محدوديتها

بلعلت محدوديت در زمان و دسترسي به مراجعان در سالن انتظار

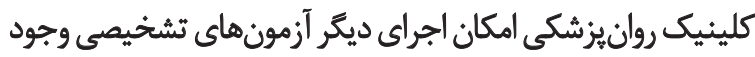

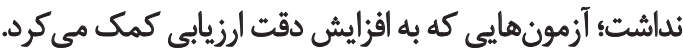

Dolesing

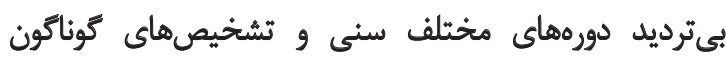

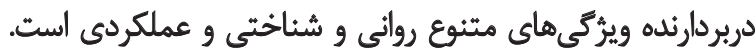

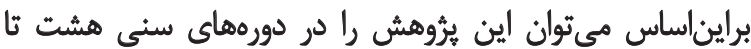

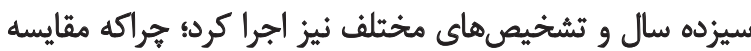

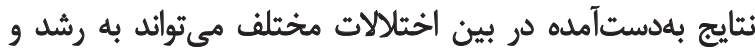

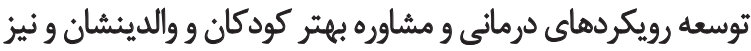

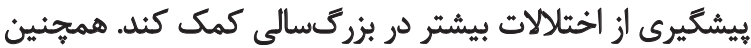

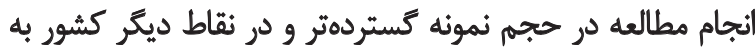

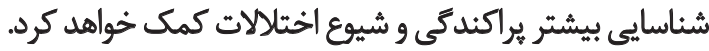

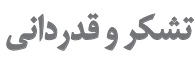

اين ثيروهش با حمايت مالي كميته تحقيقات دانشجويى دانشكاه

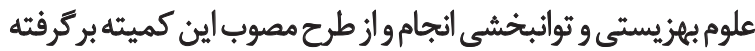

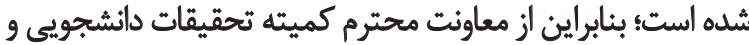

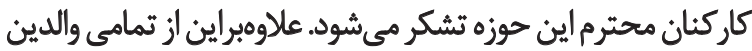

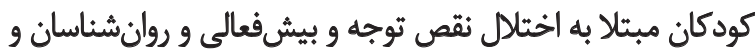

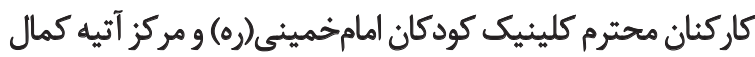
تشكر و قدردانى مى شود كه در انجام اين ثُروهش ما را يارى كردند. 
[14] Sadeghi Amrovabady Z, Esteki M, Pishyareh E, Haghgoo HA. Effect of craniosacral therapy on students' symptoms of attention deficit hyperactivity disorder. Iranian Rehabilitation Journal. 2013; 11:27-33

[15] Valizadeh S. The effect of anger management skills training on reducing of aggression in mothers of children's with Attention Deficit Hyperactive Disorder (ADHD). Iranian Rehabilitation Journal. 2010; 8(11):29-33.

[16] Brown I, Percy ME, Felce D. A comprehensive guide to intellectual and developmental disabilities. Oxford: Brookes Publication; 2007.

[17] Gershon JA. Meta-analytic review of gender differences in ADHD. Journal of Attention disorders. 2002; 5(3):143-54. doi: $10.1177 / 108705470200500302$

[18] Akhavan Karbasi S, Golestan M, Fallah R, Sadr Bafghi M. [Prevalence of attention deficit hyperactivity disorder in 6 year olds of Yazd City (Persian)]. Journal of Shahid Sadoughi University of Medical Sciences. 2008; 15(4):29-34.

[19] Hebrani P, Alaghband Rad J, Mohammadi M. [Psychiatric Disorders in Families of ADHD Children (Persian)]. Iranian Journal of Psychiatry and Clinical Psychology. 2003; 9(1):12-19.

[20] Alaghbandrad J, Moumeni F. [The association between Attention-Deficit Hyperactivity Disorder (ADHD) and learning disorder in boys aged 7-12 years old (Persian)]. Iranian Journal of Psychiatry \& Clinical Psychology. 2000; 6(2-3):32-37.

[21] Hemmati S, Amiri N, Teymouri R, Garib M. Co-Morbidity of Attention Deficit Hyperactivity Disorder (ADHD) and Tourette Syndrome in child referral psychiatry clinic in Iran. Iranian Rehabilitation Journal. 2011; 9:63-65.

[22] Ziaoddini H, Shafizadeh N. [The Epidemiology of Attention Deficit Hyperactivity Disorder and Conduct Disorder in Elementary School children in the City of Sirjan (Persian)]. Iranian Journal of Psychiatry \& Clinical Psychology. 2006;11(4):419-25.

[23] Rajabi G. [Normalizing the Raven coloure progressive matrices test on students of City Ahvaz (Persian)]. Contemporary Psychology. 2008; 3(1):23-32.

[24] Kazlaskaite V, Lynn R. Two-year test-retest reliability of the colored progressive matrices. Perceptual \& Motor Skills. 2002; 95(2):339-53. doi: 10.2466/pms.2002.95.2.354

[25] Mohammad-Esmaiil E, Alipoor A. [A preliminary study of reliability and validity and to determine cut-off points of failure CSI (CSI-4) (Persian)]. Journal of Exceptional Children. 2002; 5:239-54.

[26] Nolan EE, Gadow KD, Sprafkin J. Teacher reports of DSM-IV $\mathrm{ADHD}, \mathrm{ODD}$, and CD symptoms in schoolchildren. Journal of the American Academy of Child \& Adolescent Psychiatry. 2001; 40(2):241-49. doi: 10.1097/00004583-200102000-00020

[27] Newcorn JH, Halperin JM, Jensen PS, Abikoff HB, Arnold LE, Cantwell DP, et al. Symptom profiles in children with ADHD: Effects of comorbidity and gender. Journal of the American Academy of Child \& Adolescent Psychiatry. 2001; 40(2):137-46. doi: 10.1097/00004583-200102000-00008

[28] Cunningham CE, Boyle MH. Preschoolers at risk for attention-deficit hyperactivity disorder and oppositional defiant disorder: Family, parenting, and behavioral correlates. Journal of Abnormal Child Psychology. 2002; 30(6):555-69. doi: 10.1023/A:1020855429085

[29] Gillberg C, Gillberg I, Rasmussen P, Kadesjö B, Söderström H, Råstam M, et al. Co-existing disorders in $\mathrm{ADHD}$-implications for diagnosis and intervention. European Child \& Adolescent Psychiatry. 2004; 13:80-92. doi: 10.1007/s00787-004-1008-4

[30] Kadesjö B, Gillberg C. The comorbidity of ADHD in the general population of Swedish school-age children. Journal of Child Psychology and Psychiatry. 2001; 42(4):487-92. doi: 10.1017/ s0021963001007090

[31] Kadesjö C, Hägglöf B, Kadesjö B, Gillberg C. Attention-deficit-hyperactivity disorder with and without oppositional defiant disorder in 3 to 7 year old children. Developmental Medicine \& Child Neurology. 2003; 45(10):693-99. doi: 10.1017/ s0012162203001282

[32] Levy F, Hay DA, Bennett KS, McStephen M. Gender differences in ADHD subtype comorbidity. Journal of the American Academy of Child \& Adolescent Psychiatry. 2005; 44(4):368-76. doi: 10.1097/01.chi.0000153232.64968.c1 